\title{
Bridge to lung transplantation using short-term ambulatory extracorporeal membrane oxygenation
}

\author{
Abeel A. Mangi, MD, David P. Mason, MD, James J. Yun, MD, PhD, Sudish C. Murthy, MD, PhD, and \\ Gosta B. Pettersson, MD, PhD, Cleveland, Ohio
}

Severe respiratory failure refractory to mechanical ventilation can occur in patients awaiting lung transplantation (LTx). Use of extracorporeal membrane oxygen (ECMO) to bridge these patients to LTx is associated with considerable morbidity and mortality. ${ }^{1}$ Most techniques rely on femoral cannulation, thereby immobilizing patients. ${ }^{2}$ This in turn can lead to rapid deconditioning and predisposes patients to nosocomial pneumonia, deep venous thrombosis, and muscle wasting. ${ }^{3}$ This case highlights the use of upper-extremity ECMO, which was established without intubation in a critically ill transplant candidate, allowed ambulation, and ultimately served as a successful bridge to LTx.

\section{CLINICAL SUMMARY}

A 34-year-old man presented with dyspnea and was diagnosed with usual interstitial pneumonitis. His lung function was stable for the previous 3 years and then declined precipitously. This prompted referral to the Cleveland Clinic for expedited LTx evaluation. On presentation, diagnostic studies demonstrated a forced expiratory volume at $1 \mathrm{sec}-$ ond of $53 \%$ of predicted, diffusion lung capacity for carbon monoxide of $13 \%$ of predicted, and arterial oxygen tension of $60 \mathrm{~mm} \mathrm{Hg}$ on $8 \mathrm{~L} / \mathrm{min}$ of oxygen. Arterial oxygen desaturated quickly to $52 \%$ on a 6 -minute walk. Right-sided heart catheterization revealed a pulmonary arterial pressure of 71/29 mm Hg. During the course of the workup, further respiratory deterioration necessitated admission to the medical intensive care unit with $100 \%$ high-flow oxygen and bilevel positive-airway pressure support. Despite this, oxygen saturations could be maintained at only $80 \%$. The patient was listed for urgent LTx with a lung allocation score of 92.5 .

Because of refractory hypoxemia, severe pulmonary hypertension, and right ventricular dysfunction refractory to inhaled nitric oxide and milrinone therapy, concern of cardio-

\footnotetext{
From the Department of Thoracic and Cardiovascular Surgery, Heart and Vascular Institute, Cleveland Clinic, Cleveland, Ohio.

Disclosures: None.

Received for publication July 13, 2009; accepted for publication Feb 20, 2010; available ahead of print April 12, 2010.

Address for reprints: David P. Mason, MD, Department of Thoracic and Cardiovascular Surgery, Heart and Vascular Institute, Cleveland Clinic, 9500 Euclid Avenue/ Desk J4-1, Cleveland, OH 44195 (E-mail: masond2@ccf.org).

J Thorac Cardiovasc Surg 2010;140:713-5

$0022-5223 / \$ 36.00$

Copyright (C) 2010 by The American Association for Thoracic Surgery

doi:10.1016/j.jtcvs.2010.02.029
}

respiratory collapse on induction of anesthesia for intubation was raised. There was no patent foramen ovale on echocardiography. Consequently, the decision was made to place the patient on peripheral ECMO using sedation and local anesthesia. Veno-arterial ECMO was chosen over veno-venous to unload his right ventricle and to prevent shunting through the pulmonary vasculature. An upper-extremity approach was chosen to facilitate ambulation and maintain global fitness. In the operating room, sudden hemodynamic instability necessitated rapid institution of ECMO through the femoral vessels because of easy access. With extracorporeal support instituted, exposure of the left axillary vessels (in this right-handed patient) was possible. An $18 \mathrm{~F}$ venous cannula was placed into the right atrium via the axillary vein for drainage, and arterial return was established with a $14 \mathrm{~F}$ arterial cannula. A Bio-Medicus 560 centrifugal pump (Medtronic, Inc, Minneapolis, MN) was used with a QUADROX D membrane oxygenator (Maquet Cardiovascular, Wayne, NJ), and flow of $3.7 \mathrm{~L} / \mathrm{min}$ was established.

Two days later, ECMO flows decreased to less than 2.0 $\mathrm{L} / \mathrm{min}$ with a resultant decrease in the patient's peripheral oxygen saturation. The lack of chatter in the venous line and high line pressures in the arterial line suggested an occlusive process on the arterial side of the circuit. The patient was urgently brought to the operating room for revision of the arterial limb. The left arterial cannula was converted to an 8-mm polyethylene terephthalate graft sewn and transferred to the right axillary artery, with marked improvement in flows to as high as $4.5 \mathrm{~L} / \mathrm{min}$ and $100 \%$ peripheral oxygen saturation. The patient was regularly transferred out of bed (Figure 1) and ambulated in the intensive care unit. The patient was maintained on bilevel positive airway pressure for the first day to prevent atelectasis, after which he was placed on nasal cannula with intermittent incentive spirometry. While the patient was on ECMO, thrombocytopenia and a resultant hematoma in the right axillary cut-down site developed, which eventually stabilized. After 3 days of ECMO support, the patient underwent double LTx. The patient was slow to wean from the ventilator and required temporary tracheostomy on posttransplant day 14 . In addition, his axillary hematoma site developed necrosis that required operative debridement but was resolved with local wound care. His tracheostomy was removed, and he was discharged to a rehabilitation facility on postoperative day 31. Two years after transplant, he enjoys both excellent graft function and quality of life. 


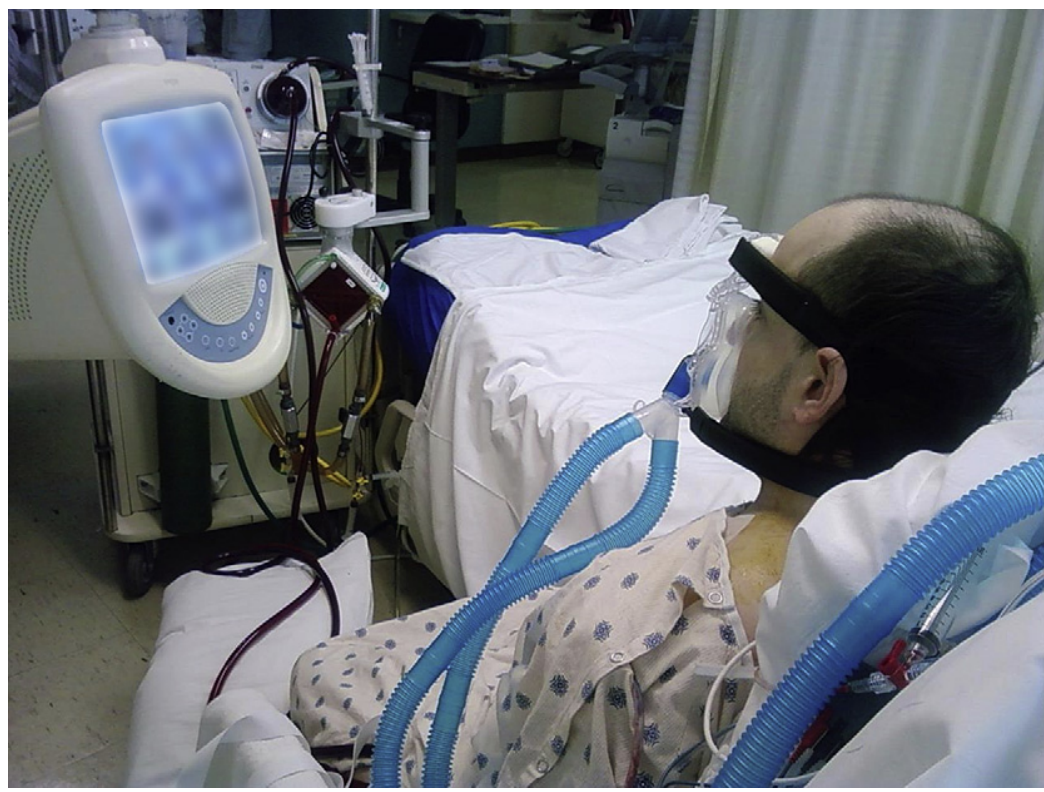

FIGURE 1. Patient out of bed and awake with ECMO support.

\section{DISCUSSION}

Bridging patients to LTx using ECMO support represents maximal therapy for severe respiratory failure. These patients are at high risk for nosocomial infection, particularly ventilator-acquired pneumonia. In addition, immobilization and ventilator dependence rapidly lead to diaphragmatic weakness and global deconditioning. ${ }^{4,5}$ LTx in this setting has been associated with poor survival. ${ }^{6}$ In an effort to avoid these negative side effects and improve survival, we instituted ECMO via the upper extremities to allow ambulation in a patient with severe respiratory failure but without intubation. We showed that this is feasible, and our patient successfully underwent transplantation.

There have been other reports of extracorporeal bridge to LTx in nonintubated patients. However, a pumpless extracorporeal lung-assist device with access through the femoral vessels for carbon dioxide clearance has been used in most cases, leaving patients bedridden. ${ }^{7,8}$ Pumpless paracorporeal support has also been used in an ambulatory fashion with central cannulation, although this requires sternotomy or thoracotomy. ${ }^{9}$ Although our technique requires a pump to provide flow adequate for oxygenation, it avoids central cannulation and can be instituted without general anesthesia. The technique is simple and reproducible. Our experience suggests that arterial cannulation should preferably be performed with a polyethylene terephthalate side graft to optimize flow and prevent potential ischemic limb complications.

\section{CONCLUSIONS}

Clearly, this technique has limitations. Although ambulation with the circuit is feasible, it is cumbersome and requires multiple support personnel for safe mobilization. Fortunately for this patient, donor organs became available after only 3 days. Feasibility of this approach for longterm support is not certain, although we were prepared to continue this therapy for as long as necessary to obtain donor lungs, precluding a major complication. Future innovations might use a more compact and transportable pump or a pumpless system. Finally, although the patient was bridged to LTx without intubation, his postoperative course was still long and complex, and it is not clear that his outcome was better than if he had been intubated before transplantation. He still required prolonged ventilatory support after transplantation, reflecting the debilitating effects of severe respiratory failure and the challenges that these high-risk patients present. Future investigation will be required to identify the optimal treatment strategy.

\section{References}

1. Jackson A, Cropper J, Pye R, Junius F, Malouf M, Glanville A. Use of extracorporeal membrane oxygenation as a bridge to primary lung transplant: 3 consecutive, successful cases and a review of the literature. J Heart Lung Transplant. 2008;27: 348-52.

2. Broome M, Palmer K, Schersten H, Frenckner B, Nilsson F. Prolonged extracorporeal membrane oxygenation and circulatory support as bridge to lung transplant. Ann Thorac Surg. 2008;86:1357-60.

3. Spicher JE, White DP. Outcome and function following prolonged mechanical ventilation. Arch Intern Med. 1987;147:421-5.

4. Wust RC, Degens H. Factors contributing to muscle wasting and dysfunction in COPD patients. Int J Chron Obstruct Pulmon Dis. 2007;2:289-300.

5. Enright S, Chatham K, Ionescu AA, Unnithan VB, Shale DJ. The influence of body composition on respiratory muscle, lung function and diaphragm thickness in adults with cystic fibrosis. J Cyst Fibros. 2007;6:384-90.

6. Mason DP, Thuita L, Nowicki ER, Murthy SC, Pettersson GB, Blacsktone EH. Should lung transplantation be performed for patients on mechanical respiratory support? The US experience. J Thorac Cardiovasc Surg. 2010;139:767-73.e1. 
7. Fischer S, Simon AR, Welte T, Hoeper MM, Meyer A, Tessmann R, et al. Bridge to lung transplantation with the novel pumpless interventional lung assist device NovaLung. J Thorac Cardiovasc Surg. 2006;131:719-23.

8. Fischer S, Hoeper MM, Tomaszek S, Simon A, Gottlieb J, Welte T, et al. Bridge to lung transplantation with the extracorporeal membrane ventilator No- valung in the veno-venous mode: the initial Hannover experience. Asaio $J$ 2007;53:168-70.

9. Schmid C, Philipp A, Hilker M, Arlt M, Trabold B, Pfeiffer M, et al. Bridge to lung transplantation through a pulmonary artery to left atrial oxygenator circuit. Ann Thorac Surg. 2008;85:1202-5.

\section{Direct aortic access through right minithoracotomy for implantation of self-expanding aortic bioprosthetic valves}

Giuseppe Bruschi, MD, ${ }^{\mathrm{a}}$ Federico De Marco, MD, ${ }^{\mathrm{a}}$ Pasquale Fratto, MD, ${ }^{\mathrm{a}}$ Jacopo Oreglia, MD, ${ }^{\mathrm{a}}$ Paola Colombo, MD, PhD, ${ }^{\mathrm{a}}$ Roberto Paino, MD ${ }^{\mathrm{b}}$ Silvio Klugmann, MD, ${ }^{\mathrm{a}}$ and Luigi Martinelli, MD, ${ }^{\mathrm{a}}$ Milan, Italy

The development of transcatheter aortic valve implantation (TAVI) for the treatment of severe aortic stenosis (AS) offers a viable option for patients at high risk or with contraindications for standard cardiac surgery. ${ }^{1}$ We report our experience of a novel surgical approach using direct aortic access through a right minithoracotomy for implanting a self-expanding aortic valve bioprosthesis (CoreValve, Medtronic-CV Luxembourg S.a.r.l.).

\section{CLINICAL SUMMARY}

Patient 1 is an 83-year-old woman (height, $145 \mathrm{~cm}$; weight, $40 \mathrm{~kg}$; body mass index, $19 \mathrm{~kg} / \mathrm{m}^{2}$ ) with symptomatic severe AS and New York Heart Association class III heart failure. She had a peak transvascular pressure gradient of $130 \mathrm{~mm} \mathrm{Hg}$, an aortic valve area of $0.5 \mathrm{~cm}^{2}$, and an aortic annulus of $18 \mathrm{~mm}$.

Patient 2 is a 60-year-old woman (height, $151 \mathrm{~cm}$; weight, $135 \mathrm{~kg}$; body mass index, $59.2 \mathrm{~kg} / \mathrm{m}^{2}$ ) with symptomatic severe AS, New York Heart Association class III, and severe bronchopneumopathy. The patient had a peak transvascular pressure gradient of $137 \mathrm{~mm} \mathrm{Hg}$ and an aortic valve area of $0.6 \mathrm{~cm}^{2}$.

According to our institutional protocol, the patients were jointly evaluated by a cardiovascular team, and the final decision of eligibility for undergoing TAVI was made. The femoral approach and axillary access were excluded because of

\footnotetext{
From the A De Gasperis Cardiology and Cardiac Surgery Department, ${ }^{\mathrm{a}}$ and Cardiothoracic Anesthesia and Intensive-care, ${ }^{\mathrm{b}}$ Niguarda Ca' Granda Hospital, Milan, Italy. Disclosures: None.

Received for publication Jan 18, 2010; accepted for publication Feb 20, 2010; available ahead of print April 15, 2010.

Address for reprints: Giuseppe Bruschi, MD, A De Gasperis Cardiology \& Cardiac Surgery Department, Niguarda Ca' Granda Hospital, Piazza dell'Ospedale Maggiore 3, 20162 Milan, Italy (E-mail: giuseppe.bruschi@fastwebnet.it).

J Thorac Cardiovasc Surg 2010;140:715-7

0022-5223/\$36.00

Copyright (c) 2010 by The American Association for Thoracic Surgery

doi:10.1016/j.jtcvs.2010.02.030
}

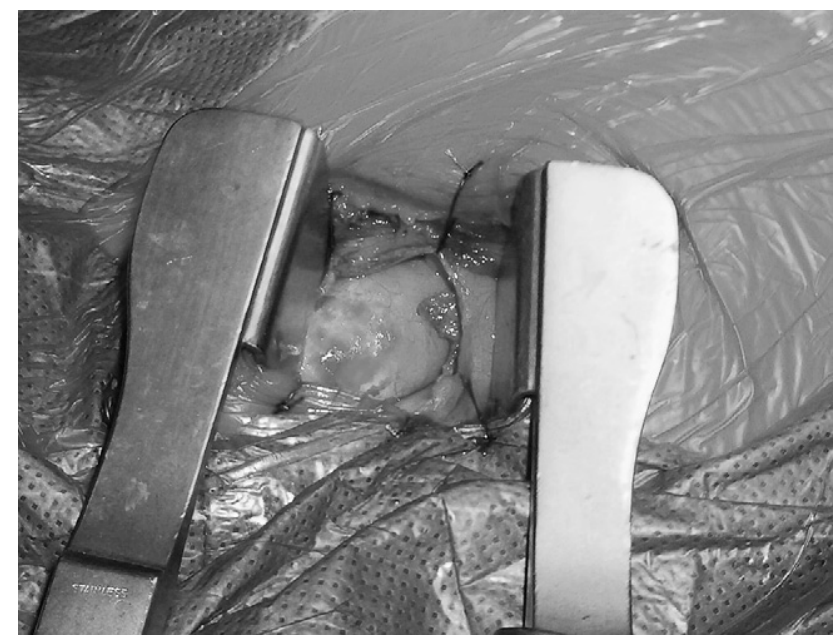

FIGURE 1. Ascending aorta exposure via right minithoracotomy

small vessel size. The transapical approach was deemed to be suboptimal because of a small-sized left ventricle (LV) and extreme LV hypertrophy (patient 1), and the difficulties in the surgical approach and suboptimal angle between the LV and the ascending aorta (patient 2). In these patients, therefore, we opted for the direct exposure of the ascending aorta via a right anterior minithoracotomy. All patients signed informed, written consent. The procedures were performed by the cardiovascular team composed of interventional cardiologists, cardiac surgeons with expertise in hybrid procedures, and cardiac anesthesiologists. The patients were under general anesthesia and mechanical ventilation. A 5.0-cm incision was made at the level of the second right anterior intercostal space with a right internal thoracic artery-sparing procedure. A soft-tissue rectractor and a small rib retractor (patient 2) were used to access the pericardium (Figure 1). A temporary pacing lead was advanced into the right ventricle, and a $6 \mathrm{~F}$ pigtail catheter was inserted for hemodynamic monitoring and landmark aortic angiography. Heparin was administered 